\title{
Parametrization and distillability of three-qubit entanglement
}

\author{
Todd A. Brun* and Oliver Cohen ${ }^{\dagger}$ \\ Physics Department, Carnegie Mellon University, \\ Pittsburgh, PA 15213
}

\begin{abstract}
There is an ongoing effort to quantify entanglement of quantum pure states for systems with more than two subsystems. We consider three approaches to this problem for three-qubit states: choosing a basis which puts the state into a standard form, enumerating "local invariants," and using operational quantities such as the number of maximally entangled states which can be distilled. In this paper we evaluate a particular standard form, the Schmidt form, which is a generalization of the Schmidt decomposition for bipartite pure states. We show how the coefficients in this case can be parametrized in terms of five physically meaningful local invariants; we use this form to prove the efficacy of a particular distillation technique for GHZ triplets; and we relate the yield of GHZs to classes of states with unusual entanglement properties, showing that these states represent extremes of distillability as functions of two local invariants.

03.67.-a 03.65.-w 03.67.Dd
\end{abstract}

Typeset using REVTEX

${ }^{*}$ Current address: Institute for Advanced Study, Einstein Drive, Princeton, NJ 08540. Email: tbrun@ias.edu

†Email: ocohen@andrew.cmu.edu 


\section{INTRODUCTION}

The importance of quantum entanglement, both as a resource for quantum information processing and as a ubiquitous feature of quantum systems, has become increasingly apparent over the last few years [1] 3]. Recent developments in quantum information theory, in particular, have stimulated interest in the quantification and manipulation of entanglement.

For bipartite pure states an essentially complete theory of entanglement now exists [3 -5], though the situation for mixed states is less definite [6]. All descriptions of bipartite pure state entanglement start with the Schmidt decomposition. It is possible to find orthonormal bases $\left\{|i\rangle_{A}\right\}$ and $\left\{|i\rangle_{B}\right\}$ for systems A and B such that we can write the joint state of the system in the form

$$
\left|\Psi_{A B}\right\rangle=\sum_{i} \sqrt{p_{i}}|i\rangle_{A} \otimes|i\rangle_{B}, \quad p_{i}>0, \quad \sum_{i} p_{i}=1 .
$$

These Schmidt coefficients $\left\{p_{i}\right\}$ are uniquely defined by the state $\left|\Psi_{A B}\right\rangle$, and are equal to the eigenvalues of the reduced density matrix $\rho_{A}$ (or equivalently, of $\rho_{B}$ ); the bases $\left\{|i\rangle_{A}\right\}$ and $\left\{|i\rangle_{B}\right\}$ are eigenbases of $\rho_{A}$ and $\rho_{B}$, respectively, so the local density matrices are diagonal in this choice of bases. This choice of bases also minimizes the number of terms needed to represent $\left|\Psi_{A B}\right\rangle$.

For tripartite or multipartite states, there is no equivalent to the Schmidt decomposition (11) [7]). Three main approaches to parametrizing tripartite or multipartite entanglement have been followed so far. First, one may choose the local bases to put the joint state into a standard form. Often these standard forms are intended to generalize some aspect of the Schmidt decomposition in the bipartite case [8 [15]. Second, one may try to identify a complete set of locally invariant quantities, functions of the state which are invariant under local unitary transformations [8, 12,16 [19], and which uniquely characterize equivalent states. The coefficients of a standard form are obviously such quantities, but they may not have readily meaningful physical interpretations. Third, one may identify operational quantities, such as the number of Greenberger-Horne-Zeilinger (GHZ) triplets or EPR pairs that can be distilled from the state by some procedure [11,21,20].

In section II we consider a number of proposals for standard forms of three-qubit pure states, concentrating especially on those which generalize some aspect of the bipartite Schmidt decomposition: the minimal form [13, 15], the two-term form [13,22, 21], and the Schmidt form. This form was given briefly in [11] and independently in [12. In section III we examine it in greater detail. We give an explicit parametrization of the coefficients in terms of five locally invariant quantities, and discuss their physical significance.

We make use of the Schmidt form to prove analytically the reliability of a proposed distillation technique for GHZ triplets from general three-qubit pure states [11]; we present this proof in section IV.

In [8] Linden and Popescu proposed characterizing the entanglement properties of threequbit states by examining the "orbits" of the states under general local unitary transformation. This was carried a step further by Carteret and Sudbery [19], who proved that most states have a certain generic behavior under such transformations, but identified classes of 'special' states which they speculated to have unusual entanglement properties. In section $\mathrm{V}$ we briefly review these 'special' states, then analytically evaluate the yield of GHZs under 
the distillation protocol of [11] and section IV. By examining the yield of these states as a function of the invariant parameters from section III, and also of the locally invariant "residual tangle" $\tau_{A B C}$ of Coffman, Kundu and Wootters [18], we verify that these classes of states are indeed exceptional by this operational measure, representing extremes of distillability or undistillability. We briefly compare the results using this protocol to the recently discovered optimal distillation method of [21], and find that they are entirely consistent. Our conclusions are summarized in section VI.

\section{REVIEW OF STANDARD FORMS FOR THREE-QUBIT STATES}

Two qubits can always be represented in their Schmidt decomposition (四)

$$
|\psi\rangle=\sqrt{p}|00\rangle+\sqrt{1-p}|11\rangle,
$$

characterized by a single Schmidt coefficient $p$ (or equivalently $1-p$ ). Without loss of generality, we adopt the convention that $p \geq 1 / 2$ and the corresponding eigenvector is $|0\rangle$.

Most attempts to define a standard form for a three-qubit state attempt either to generalize some property of the bipartite Schmidt decomposition, or make use of the Schmidt decomposition between one of the bits and the other two, or both. For instance, we can make a Schmidt decomposition between qubit A and qubits B and C, writing the three-qubit state in the form

$$
|\psi\rangle=\sqrt{p}|0\rangle_{A}\left|\psi_{0}\right\rangle_{B C}+\sqrt{1-p}|1\rangle_{A}\left|\psi_{1}\right\rangle_{B C}
$$

Choosing the Schmidt basis for qubit A guarantees that the correlated states of qubits B and $\mathrm{C}$ must be orthogonal: $\left\langle\psi_{0} \mid \psi_{1}\right\rangle=0$.

The sixteen real parameters to describe a generic pure state of three qubits can be reduced to fifteen by normalization, and to five which are invariant under the ten-dimensional group of local unitary transformations [8,19]; unfortunately, no single choice of five quantities has proven completely satisfactory. One simple parametrization that has been proposed [ 8 10] is the Linden-Popescu-Schlienz (LPS) standard form. One begins with a state in form (3). One can then choose one of the two correlated states, say $\left|\psi_{0}\right\rangle_{B C}$, and find its corresponding Schmidt bases. The resulting state for the three qubits has the form

$$
\begin{aligned}
|\psi\rangle= & \sqrt{p}|0\rangle_{A}\left(a|00\rangle_{B C}+\sqrt{1-a^{2}}|11\rangle_{B C}\right) \\
& +\sqrt{1-p}|1\rangle_{A}\left(\gamma\left(\sqrt{1-a^{2}}|00\rangle_{B C}-a|11\rangle_{B C}\right)+f|01\rangle_{B C}+g|10\rangle_{B C}\right),
\end{aligned}
$$

where $p, a$ and $f$ are real positive numbers, $g$ is complex, and $\gamma=\left(1-f^{2}-|g|^{2}\right)^{1 / 2}$. Together these give five independent real parameters.

The vectors $\left|\psi_{0}\right\rangle_{23}$ and $\left|\psi_{1}\right\rangle_{23}$ span a two-dimensional subspace of the Hilbert space for qubits 2 and 3. It's possible to make an interesting variation on the LPS idea using a result of Niu and Griffiths, who showed [24] that any such two-dimensional subspace can be given basis vectors of the form

$$
\begin{aligned}
& \left|\chi_{0}\right\rangle=\sqrt{q}|00\rangle_{23}+\sqrt{1-q}|11\rangle_{23}, \\
& \left|\chi_{1}\right\rangle=\sqrt{r}|01\rangle_{23}+\sqrt{1-r}|10\rangle_{23},
\end{aligned}
$$


for some choice of a product basis for the 4-D Hilbert space of the two bits, where $q$ and $r$ are real numbers between 0 and 1 . Using this basis leads to a unique standard form

$$
\begin{aligned}
|\psi\rangle= & \sqrt{p}|0\rangle(a \sqrt{q}|00\rangle+a \sqrt{1-q}|11\rangle+b \sqrt{r}|01\rangle+b \sqrt{1-r}|10\rangle) \\
& +\sqrt{1-p}|1\rangle\left(-b^{*} \sqrt{q}|00\rangle-b^{*} \sqrt{1-q}|11\rangle+a \sqrt{r}|01\rangle+a \sqrt{1-r}|10\rangle\right),
\end{aligned}
$$

where $a$ is real and $a^{2}+|b|^{2}=1$. This then gives five independent real parameters: $p, q, r$, $a$, and the phase of $b$. This form treats the $|0\rangle$ and $|1\rangle$ terms more symmetrically than LPS; however, there is still a lack of symmetry under interchange of the bits.

More interesting from a fundamental point of view are attempts to generalize some aspect of the Schmidt decomposition. Three such properties suggest themselves. First, the Schmidt decomposition is the choice of orthonormal bases for the local Hilbert spaces which minimizes the number of terms needed to represent the state. Second, any two qubit state can be written as the sum of only two product vectors. (For $N$-dimensional systems, $N$ product vectors are needed.) Third, the Schmidt decomposition diagonalizes the reduced density matrices of the local subsystems. No single representation for tripartite systems has all three properties, but they can be generalized individually.

Acín et al. [13] have shown that all three-qubit states can be written in the form

$$
|\psi\rangle=\lambda_{0}|000\rangle+\lambda_{1} \mathrm{e}^{i \phi}|100\rangle+\lambda_{2}|101\rangle+\lambda_{3}|110\rangle+\lambda_{4}|111\rangle
$$

by a suitable choice of basis, where the $\lambda_{i}$ are all real and positive and $\phi$ is a phase between 0 and $\pi$. With only five terms, this is a minimal description, and in that sense a generalization of the bipartite Schmidt decomposition. A similar form has been described by Higuchi and Sudbery [14],

$$
|\psi\rangle=\lambda_{0} \mathrm{e}^{i \phi}|000\rangle+\lambda_{1}|100\rangle+\lambda_{2}|010\rangle+\lambda_{3}|001\rangle+\lambda_{4}|111\rangle
$$

which has the added benefit of being symmetric under interchange of the qubits. Carteret, Higuchi and Sudbery [15] have shown how to generalize this construction to give a unique minimal representation for systems of any dimension. These minimal forms have practical benefits: with a small number of terms, they can simplify the calculation of locally invariant quantities 23. However, the $\lambda_{i}$ and $\phi$ themselves have no obvious physical interpretation.

This minimal property can be generalized in another way, by relaxing the requirement that the product vectors be orthogonal. Acín et al. and Dür, Vidal and Cirac have also shown [13,22] that almost all three qubit states can be written in the form

$$
\left|\Psi_{A B C}\right\rangle=\mu_{1}\left|a_{1} b_{1} c_{1}\right\rangle+\mu_{2} \mathrm{e}^{i \phi}\left|a_{2} b_{2} c_{2}\right\rangle,
$$

where the vectors are normalized but not orthogonal. There are six real parameters, $\mu_{1}, \mu_{2}$, $\left\langle a_{1} \mid a_{2}\right\rangle,\left\langle b_{1} \mid b_{2}\right\rangle,\left\langle c_{1} \mid c_{2}\right\rangle$ and $\phi$; imposing normalization reduces this to five.

Interestingly, not all three qubit states can be written in the form (9); a small subclass of states require a minimum of three product terms [13. Dür, Vidal and Cirac made use of this result to prove that there are two classes of three-qubit pure states which cannot be interconverted with nonzero probability [22]. The class that requires three terms is a three-parameter family, and is characterized by vanishing residual tangle $\tau_{A B C}=0$ [18] (see 
section V). Acín, Dür and Vidal also used this form to demonstrate a method of converting a single copy of a three qubit state into a GHZ triplet with maximum probability [21].

The third generalization is to find bases for all three qubits which diagonalize their reduced density matrices. That is, one can simultaneously put each bit in its Schmidt decomposition with respect to the other two. This form was proposed in [11] and independently in [12]. The state has the form

$$
\begin{aligned}
|\psi\rangle= & a|000\rangle+b|001\rangle+c|010\rangle+d|011\rangle \\
& +e|100\rangle+f|101\rangle+g|110\rangle+h|111\rangle,
\end{aligned}
$$

which looks just like a generic three-qubit state with 16 parameters. However, using each of the three qubits in turn we can write $|\psi\rangle$ in a form similar to (3), with orthogonality conditions which impose restrictions on the possible values of the coefficients in (10). We can use these relationships to reduce these coefficients to five independent parameters, as we show in the next section.

\section{PARAMETRIZING THE SCHMIDT FORM}

By redefining the relative phases of the basis vectors

$$
|0\rangle_{j},|1\rangle_{j} \rightarrow \exp \left(i \phi_{j}\right)|0\rangle, \exp \left(i \theta_{j}\right)|1\rangle
$$

we can choose to make four of the coefficients real. A convenient choice is to make $a, d, f, g$ real, while $b, c, e, h$ remain complex. The state must also be normalized, which imposes the condition

$$
a^{2}+|b|^{2}+|c|^{2}+d^{2}+\left|e^{2}\right|+f^{2}+g^{2}+|h|^{2}=1 .
$$

This leaves 11 undetermined parameters.

We can now express the larger eigenvalues $p_{A, B, C}$ of the reduced density matrices $\rho_{A, B, C}$ in terms of the coefficients:

$$
p_{A}=a^{2}+|b|^{2}+|c|^{2}+d^{2}, \quad \text { etc. }
$$

(the smaller eigenvalues obviously being $1-p_{A, B, C}$ ). Finally, the states $\left|\psi_{0,1}\right\rangle_{k l}$ correlated with basis vectors $|0\rangle_{j}$ and $|1\rangle_{j}$ must be orthogonal to each other. This gives three more equations:

$$
a e^{*}+b f+c g+d h^{*}=0, \text { etc. }
$$

Because these equations are complex, they are equivalent to six real equations.

Combining these restrictions, we now have fourteen equations in sixteen unknowns. Thus, in addition to the eigenvalues $p_{A, B, C}$ we would expect there to be two more free parameters. Can we identify reasonable candidates for these parameters? It turns out that natural choices are the two probabilities $a^{2}$ and $|h|^{2}$. These parameters are symmetric under interchanges of the three qubits, and have a fairly simple physical interpretation: they are the probabilities of all three qubits giving the same result ( 0 or 1 , respectively) when measured in their 
Schmidt bases. Moreover, the coefficients of the other state vectors can all be calculated in terms of the five probabilities $a^{2},|h|^{2}$, and $p_{A, B, C}$, up to a sign.

Define $p_{\text {sum }}=p_{A}+p_{B}+p_{C}$. The expressions for the norms of the coefficients are then simple:

$$
\begin{aligned}
|b|^{2},|c|^{2},|e|^{2} & =\frac{\left(2 p_{C, B, A}-1\right)|h|^{2}-\left(p_{\mathrm{sum}}-p_{C, B, A}-1\right)\left(2 a^{2}-p_{\mathrm{sum}}+1\right)}{2 p_{\mathrm{sum}}-3} \\
d^{2}, f^{2}, g^{2} & =\frac{\left(2 p_{A, B, C}-1\right) a^{2}-\left(p_{\mathrm{sum}}-p_{A, B, C}-1\right)\left(2|h|^{2}+p_{\mathrm{sum}}-2\right)}{2 p_{\mathrm{sum}}-3} .
\end{aligned}
$$

The phases of $b, c, e$ are more complicated. If we define the variables $\phi_{b, c, e}$ by $b=$ $|b| \exp \left(i \phi_{b}\right), c=|c| \exp \left(i \phi_{c}\right)$, and $e=|e| \exp \left(i \phi_{e}\right)$, the constraint equations (14) imply after a bit of algebra that

$$
\begin{aligned}
& \cos \left(\phi_{b}\right), \sin \left(\phi_{b}\right)=\left(Q_{1,2} /|b|\right)\left(\mp 2 a d f+g\left(a^{2}+d^{2}+f^{2}-g^{2}\right)\right), \\
& \cos \left(\phi_{c}\right), \sin \left(\phi_{c}\right)=\left(Q_{1,2} /|c|\right)\left(\mp 2 a d g+f\left(a^{2}+d^{2}-f^{2}+g^{2}\right)\right), \\
& \cos \left(\phi_{e}\right), \sin \left(\phi_{e}\right)=\left(Q_{1,2} /|e|\right)\left(\mp 2 a f g+d\left(a^{2}-d^{2}+f^{2}+g^{2}\right)\right), \\
& \cos \left(\phi_{h}\right), \sin \left(\phi_{h}\right)=\left(Q_{1,2} /|h|\right)\left(\mp 2 d f g+a\left(-a^{2}+d^{2}+f^{2}+g^{2}\right)\right),
\end{aligned}
$$

where $Q_{1}$ and $Q_{2}$ are two constants. We can solve for the values of $Q_{1}$ and $Q_{2}$ by using the identity $\sin ^{2}(\phi)+\cos ^{2}(\phi)=1$ and substituting (15) for $|b|, \ldots, g$.

In the Schmidt form for three-qubit pure states, each of the five parameters has a reasonably straightforward physical interpretation. The three parameters $p_{A}, p_{B}, p_{C}$ are the larger (i.e., $p \geq 1 / 2$ ) eigenvalues of the reduced density operators for each of the three qubits, and correspond to the probabilities of obtaining the more likely of the two possible outcomes (which by convention we label $|0\rangle$ ) when we measure each of the qubits in its Schmidt basis. These parameters are closely related to the minimum absolutely selective information [11] for each qubit, which is given by the entropy function

$$
\min S_{i}=-\left(p_{i} \log _{2} p_{i}+\left(1-p_{i}\right) \log _{2}\left(1-p_{i}\right)\right) .
$$

This quantity is the minimum amount of fundamentally unpredictable classical information generated by carrying out a measurement on qubit $i$, given a free choice of measurement basis [11]. By using the Schmidt form to choose measurement bases we can simultaneously minimize the absolutely selective information for all three qubits.

The parameters $p_{A}, p_{B}, p_{C}$ range from $1 / 2$ to 1 (since they are defined to be the larger eigenvalues of their corresponding local density matrices). Similarly, $a^{2}$ ranges from 0 to 1 , and $|h|^{2}$ from 0 to $1 / 2$. However, this does not mean that these parameters can take arbitrary values within these ranges. Some choices of parameter values correspond to no physical state, and give nonsensical values for (15) and (16).

In particular, the local probabilities must obey the triangle inequalities

$$
\begin{aligned}
& p_{A}\left(1-p_{A}\right)+p_{B}\left(1-p_{B}\right) \geq p_{C}\left(1-p_{C}\right), \\
& p_{B}\left(1-p_{B}\right)+p_{C}\left(1-p_{C}\right) \geq p_{A}\left(1-p_{A}\right), \\
& p_{C}\left(1-p_{C}\right)+p_{A}\left(1-p_{A}\right) \geq p_{B}\left(1-p_{B}\right) ;
\end{aligned}
$$

these imply, for instance, that if $p_{A}=1$ then $p_{B}=p_{C}$. The restrictions on $a^{2}$ and $|h|^{2}$ are more complicated, but they too display an interdependency in their range of values. In particular, as $p_{\text {sum }} \rightarrow 3$ we must have $a^{2} \rightarrow 1$ and $|h|^{2} \rightarrow 0$. 


\section{PROOF OF DISTILLABILITY}

The Schmidt form can provide analytical insight when addressing specific problems. For example, the efficacy of a recently proposed tripartite distillation protocol [11] can be demonstrated with its help.

Consider a state of three qubits in an arbitrary product basis, which can be written in the form (10). We can straightforwardly calculate the quantity $p_{A}\left(1-p_{A}\right)$

$$
\begin{aligned}
p_{A}\left(1-p_{A}\right)= & |a f-b e|^{2}+|a g-c e|^{2}+|a h-d e|^{2} \\
& +|b g-c f|^{2}+|b h-d f|^{2}+|c h-d g|^{2},
\end{aligned}
$$

This expression is a polynomial in the coefficients and their complex conjugates, and is correct in any basis. If the state is in the Schmidt form, this simplifies to

$$
p_{A}\left(1-p_{A}\right)=\left(a^{2}+|b|^{2}+|c|^{2}+d^{2}\right)\left(|e|^{2}+f^{2}+g^{2}+|h|^{2}\right) .
$$

Let us assume that we have written the state in Schmidt form, such that the states $\{|0\rangle,|1\rangle\}$ for each qubit $j$ are eigenstates of the local density matrix with eigenvalues $p_{j}$ and $1-p_{j}$, respectively. Suppose we now perform a weak measurement on each of the three qubits. First, allow each qubit to interact with a separate ancilla bit initially in state $|0\rangle$, such that

$$
\begin{aligned}
& |0\rangle \otimes|0\rangle_{\mathrm{anc}} \rightarrow \sqrt{1-\epsilon}|0\rangle \otimes|0\rangle_{\mathrm{anc}}+\sqrt{\epsilon}|0\rangle \otimes|1\rangle_{\mathrm{anc}} \\
& |1\rangle \otimes|0\rangle_{\mathrm{anc}} \rightarrow|1\rangle \otimes|0\rangle_{\mathrm{anc}}
\end{aligned}
$$

where $\epsilon \ll 1$. Then measure the three ancilla bits. With a probability of $\epsilon\left(p_{\text {sum }}\right)$ one will find one or more of the ancilla bits in state $|1\rangle_{\text {anc }}$, in which case the procedure has failed. Otherwise, this step has succeeded and the three qubits are now in a new state with slightly different coefficients $a^{\prime}, b^{\prime}, \ldots, h^{\prime}$. The changes in the coefficients are

$$
\begin{aligned}
\Delta a & =-(\epsilon / 2)\left(3-p_{\text {sum }}\right) a, \\
\Delta(b, c, e) & =-(\epsilon / 2)\left(2-p_{\text {sum }}\right)(b, c, e), \\
\Delta(d, f, g) & =-(\epsilon / 2)\left(1-p_{\text {sum }}\right)(d, f, g), \\
\Delta h & =(\epsilon / 2) p_{\text {sum }} h .
\end{aligned}
$$

This very simple form results because the state is in Schmidt form. After this procedure the bases for the three bits will generally no longer be the correct Schmidt basis (though it will be close to it), so the expression (20) cannot be used; but (19) is always correct. Thus we get a change in $p_{A}\left(1-p_{A}\right)$

$$
\begin{aligned}
\Delta\left[p_{A}\left(1-p_{A}\right)\right]= & -\epsilon\left(4-2\left(p_{A}+p_{B}+p_{C}\right)\right)\left(|a f-b e|^{2}+|a g-c e|^{2}\right) \\
& -\epsilon\left(3-2\left(p_{A}+p_{B}+p_{C}\right)\right)\left(|a h-d e|^{2}+|b g-c f|^{2}\right) \\
& -\epsilon\left(2-2\left(p_{A}+p_{B}+p_{C}\right)\right)\left(|b h-d f|^{2}+|c h-d g|^{2}\right) \\
= & -\epsilon\left(3-2\left(p_{A}+p_{B}+p_{C}\right)\right) p_{A}\left(1-p_{A}\right) \\
& -(\epsilon / 2)\left(|a f-b e|^{2}+|a g-c e|^{2}-|b h-d f|^{2}-|c h-d g|^{2}\right)
\end{aligned}
$$


By making use of equations (13) and (14), this expression simplifies to

$$
\begin{aligned}
\Delta\left[p_{A}\left(1-p_{A}\right)\right]= & \epsilon\left[\left(2\left(p_{A}+p_{B}+p_{C}\right)-3\right) p_{A}\left(1-p_{A}\right)\right. \\
& \left.+p_{A}\left(a^{2}-|e|^{2}+|h|^{2}-d^{2}\right)+d^{2}-a^{2}\right],
\end{aligned}
$$

which using (15) further simplifies to

$$
\begin{aligned}
\Delta\left[p_{A}\left(1-p_{A}\right)\right]= & \frac{\epsilon\left(2 p_{A}-1\right)}{2 p_{A}+2 p_{B}+2 p_{C}-3}\left[2\left(a^{2}+\left|h^{2}\right|\right)\left(p_{B}+p_{C}-1\right)\right. \\
& \left.-\left(2 p_{A}-1\right)\left(p_{A}+p_{B}+p_{C}-1\right)\left(p_{A}+p_{B}+p_{C}-2\right)\right] .
\end{aligned}
$$

The prefactor to (25) is strictly positive, as is the first term inside the brackets. The second term is positive if $p_{A}+p_{B}+p_{C}<2$; any state that satisfies this criterion will evolve towards the GHZ state and have a nonzero yield.

For $p_{A}+p_{B}+p_{C} \geq 2$, the sign of (25) depends on the relative sizes of the first and second terms inside the brackets. The last two equations of (15) show that for $p_{A}+p_{B}+p_{C} \geq 2$, the fact that $f^{2}+g^{2}>0$ implies

$$
2 a^{2}\left(p_{B}+p_{C}-1\right) \geq\left(2 p_{A}+p_{B}+p_{C}-2\right)\left(p_{A}+p_{B}+p_{C}-2\right),
$$

which yields the inequalities

$$
\begin{aligned}
& 2 a^{2}\left(p_{B}+p_{C}-1\right)-\left(2 p_{A}-1\right)\left(p_{A}+p_{B}+p_{C}-1\right)\left(p_{A}+p_{B}+p_{C}-2\right) \\
\geq & \left(2 p_{A}+p_{B}+p_{C}-2\right)\left(p_{A}+p_{B}+p_{C}-2\right) \\
& -\left(2 p_{A}-1\right)\left(p_{A}+p_{B}+p_{C}-1\right)\left(p_{A}+p_{B}+p_{C}-2\right) \\
= & \left(1-p_{A}\right)\left(p_{A}+p_{B}+p_{C}-2\right)\left(2 p_{A}+2 p_{B}+2 p_{C}-3\right) \geq 0 .
\end{aligned}
$$

This straightforwardly implies

$$
\Delta\left[p_{A}\left(1-p_{A}\right)\right] \geq \epsilon\left(2 p_{A}-1\right)\left(1-p_{A}\right)\left(p_{A}+p_{B}+p_{C}-2\right) \geq 0 .
$$

Because of the symmetry of the protocol, $p_{B}\left(1-p_{B}\right)$ and $p_{C}\left(1-p_{C}\right)$ must also increase. So one step of this protocol must move the state towards the GHZ with nonvanishing probability, and will (in general) produce a nonzero yield of GHZ triplets.

There are three circumstances in which this result can fail. First, no product state can ever be distilled to a GHZ by this method. At least one of $p_{A}, p_{B}, p_{C}$ must equal 1 in this case, which causes the rate (25) corresponding to it to vanish. This is not immediately obvious from the form of (25), but it is easily checked using (15) and (18) - if $p_{A}=1$, then $p_{B}=p_{C}=a^{2}$, and (25) is equal to zero.

Second, there are states with $p_{A}+p_{B}+p_{C}=2$ for which $a^{2}=|h|^{2}=0$, again making (25) vanish. These are a subset of the triple states discussed in section $\mathrm{V}$ below, which are equivalent to states of the form (31); these states have vanishing residual tangle. Finally, it is possible for a state with $p_{A}+p_{B}+p_{C}>2$ to evolve to one of these triple states. All such states will also have vanishing residual tangle [22], and conversely all states with vanishing residual tangle will evolve under this distillation protocol to a triple state with $p_{A}+p_{B}+p_{C}=2$, and hence have zero yield of GHZs. This can be clearly seen in Fig. 1. 


\section{ENTANGLEMENT AND DISTILLABILITY}

Linden and Popescu [8] proposed characterizing three-qubit states by the dimensions of their orbits under the action of the local unitary group. Generically, tripartite pure states of qubits have ten-dimensional orbits, equal to the dimension of the local unitary group. The very interesting results of Carteret and Sudbery [19] give a complete classification of all states for three qubits which behave nongenerically under local unitary transformations; these 'special' states have stabilizers of nonzero dimension, and hence orbits of dimension $<10$ (see [19]). This behavior suggests that these 'special' classes have unusual entanglement properties, which might be evident in other measures of entanglement.

We have numerically simulated the distillation of generic states by the algorithm described above in section IV, in order to determine the yield of GHZ triplets as a function of various parameters, especially the parameters used to describe the Schmidt form. We have also calculated analytical expressions for the yield of states in the exceptional classes enumerated by Carteret and Sudbery. We find that these states are indeed exceptional by this operational criterion, as we describe below.

Most important in calculating the yield of GHZs is the sum of the local eigenvalues $p_{\text {sum }} \equiv p_{A}+p_{B}+p_{C}$. This quantity determines the probability of failure in one step of the infinitesimal distillation procedure of section IV, with the probability of failure being $\epsilon p_{\text {sum }}$. If it takes $N$ steps to become sufficiently close to a GHZ triplet, the expected yield is

$$
Y=\prod_{n=1}^{N}\left(1-\epsilon p_{\text {sum }}(n)\right) \approx \exp \left\{-\sum_{n} \epsilon p_{\text {sum }}(n)\right\}
$$

where $p_{\text {sum }}(n)$ is the value of $p_{\text {sum }}$ at the $n$th step. In the limit of infinitesimal steps the sum inside the exponent becomes an integral. Calculating $p_{\text {sum }}(n)$ analytically is no simple matter for a general state; the equations (22) for the change in the coefficients become differential equations in the limit, but must be supplemented by an additional change of basis between steps, since in general the bases will no longer be the Schmidt basis for the new state. While this is simple to do numerically, analytically it is challenging.

Fortunately, the classes of exceptional states are generally expressible in simple forms which make it possible to integrate the equations (22) in closed form, and derive simple expressions for the yield of GHZs. Interestingly, the steps of the GHZ distillation technique commute with local unitary transformations. Because of this, the distillation procedure preserves the stabilizer of the initial state, and hence must take 'special' states to other 'special' states of the same type. This gives another way of understanding why certain special states are not distillable to GHZs.

In addition to $a^{2},|h|^{2}$, and $p_{\text {sum }}$, we looked at the dependence of the GHZ yield on one other locally invariant quantity. This is the residual tangle of Coffman et al. [18], which can be written

$$
\tau_{A B C}=2\left(\lambda_{1}^{A B} \lambda_{2}^{A B}+\lambda_{1}^{A C} \lambda_{2}^{A C}\right)
$$

where $\lambda_{1}^{i j}$ and $\lambda_{2}^{i j}$ are the (positive) eigenvalues of the matrix $\sqrt{\rho_{i j} \tilde{\rho}_{i j}}$. Here $\rho_{i j}$ is the density operator for the two-party $i j$ system, and $\tilde{\rho}_{i j}$ is the "spin-flipped" density operator: $\tilde{\rho}_{i j}=\left(\sigma_{y} \otimes \sigma_{y}\right) \rho_{i j}^{*}\left(\sigma_{y} \otimes \sigma_{y}\right)$. It has been suggested [18] that the residual tangle is a measure 
of the irreducible three-way ("GHZ-type") entanglement of a tripartite state, beyond any two-party ("EPR-type") entanglement that may be contained in such a state. As such, it is of particular interest in discussing distillability below. Also, its square $\tau_{A B C}{ }^{2}$ is a polynomial quantity, which makes it analytically tractable.

Triple States. For this set of states the residual tangle vanishes [18]. We previously described states in this set as "triple" states [11], because they are equivalent under local unitary transformations to states with just three components:

$$
\left|\psi_{\text {tr }}\right\rangle=b|001\rangle+c|010\rangle+e|100\rangle .
$$

Carteret and Sudbery [19] refer to these as "beechnut" states; they all have $p_{\text {sum }} \geq 2$. For triple states with $p_{\text {sum }}>2$ each step of the infinitesimal distillation protocol reduces $p_{\text {sum }}$, but leaves the state a triple state. If $p_{\text {sum }}=2$, the actions on the three qubits cancel out, leaving the state unchanged. States of this type have vanishing primary yield for the tripartite distillation protocols described in section IV and in [11]; indeed, Dür, Vidal and Cirac have shown that no procedure can transform one copy of a state with zero residual tangle into a GHZ with nonzero probability [22].

Because the distillation procedures of section IV and [11] preserve the classes of 'special' states, it is easy to see why they cannot produce GHZs from triple states; because all triple states have $p_{\text {sum }} \geq 2$, they cannot include the GHZ state $\left(p_{\text {sum }}=3 / 2\right)$ as a limit. The set of product states (or "bystander states" in the terminology of Carteret and Sudbery) is similarly undistillable. The result of Dür, Vidal and Cirac, however, goes beyond this, since it assumes nothing about the symmetry of the procedure.

The symmetric version of state (31) (with $b=c=e=1 / \sqrt{3}$ ) is termed by Dür, Vidal and Cirac the "W" state, and seems to fill a role for the zero residual tangle states similar to the role filled by the GHZ for all other states: it is, in some sense, maximally entangled. We will say a bit more about this below. All other 'special' classes include the GHZ as a limit, and therefore are distillable.

Generalized GHZ states. These states can be written in Schmidt form

$$
|\psi\rangle=a|000\rangle+h|111\rangle .
$$

They have $p_{A}=p_{B}=p_{C}=a^{2}$, residual tangle $\tau_{A B C}=4 a^{2} h^{2}$. A single step of the infinitesimal distillation procedure gives a new generalized GHZ with coefficients $a^{\prime}=a+\Delta a$, $h^{\prime}=h+\Delta h:$

$$
\Delta a=-(\epsilon / 2)\left(3-p_{\text {sum }}\right) a, \quad \Delta h=+(\epsilon / 2) p_{\text {sum }} h,
$$

so (29) can readily be evaluated to give the yield of GHZs

$$
Y=1-\sqrt{1-\tau_{A B C}}=(2 / 3)\left(3-p_{\text {sum }}\right) .
$$

These states are the most distillable three qubit states as a function of both $\tau_{A B C}$ and $p_{\text {sum }}$; we can see this in Figures 1 and 2 below.

Slice states. In Schmidt form these are

$$
|\psi\rangle=a|000\rangle+d|011\rangle-e|100\rangle+h|111\rangle, \quad a e=d h,
$$


plus similar states derived by permuting the order of the bits. These states have $p_{B}=p_{C}=$ $a^{2}+e^{2}, p_{A}=a^{2}+d^{2}, \tau_{A B C}=4(a h+d e)^{2}=4 a^{2}\left(h+e^{2} / h\right)^{2}$. Imposing normalization and the orthogonality condition on (35) we see that this is a two-parameter family of states. For these two parameters we may choose $a^{2}$ and $h^{2}$, or equivalently $p_{A}$ and $p_{B}$. One step of the infinitesimal distillation protocol applied to state (35) leaves qubits $\mathrm{B}$ and $\mathrm{C}$ in their Schmidt bases, but not qubit A; a change of basis must be applied to A to put the new state in Schmidt form. This new state is still a slice state, and has new parameters $p_{A}^{\prime}=p_{A}+\Delta p_{A}$, $p_{B}^{\prime}=p_{B}+\Delta p_{B}$,

$$
\begin{aligned}
\Delta p_{A} & =2 \epsilon\left(p_{A}+2 p_{B}-1\right) p_{A}, \\
\Delta p_{B} & =2 \epsilon\left(p_{A}+2 p_{B}-2\right) p_{B}-\epsilon p_{A}\left(p_{A}+p_{B}-1\right) /\left(2 p_{A}-1\right) .
\end{aligned}
$$

The yield is difficult to evaluate analytically, but numerical evidence shows that generic slice states are not extremes of distillability. With each step of the distillation protocol, the parameters $p_{B}=p_{C}$ approach $1 / 2$, but $p_{A}$ actually moves away. However, when $p_{B}=p_{C}=$ $1 / 2$, this subclass of slice states does have extremal behavior. Carteret and Sudbery term this subclass the maximal slice states.

Maximal slice or Slice-ridge states are of form (35) with $a^{2}+e^{2}=1 / 2=p_{B}=p_{C}$. This subclass is parametrized by a single number, which can be taken to be $p_{A}$. Because only $p_{A}$ is larger than $1 / 2$, there is no need to perform the GHZ distillation procedure on qubits $\mathrm{B}$ and $\mathrm{C}$; performing it on $\mathrm{A}$ alone preserves the form of the state, with

$$
\Delta p_{A}=-(\epsilon / 2) p_{A}\left(1-p_{A}\right),
$$

giving a yield of GHZs

$$
Y=1-\sqrt{1-\tau_{A B C}}=2\left(2-p_{\text {sum }}\right) .
$$

The expression for the primary yield in terms of the residual tangle is identical to that for the GHZ-type states, while in terms of $p_{\text {sum }}$ it is not. In terms of $\tau_{A B C}$ it is one of the most distillable types of state (see Fig. 1). In terms of $p_{\text {sum }}$ (Fig. 2) it appears to be one of the least distillable types of states; this is because maximal slice states have the minimum $\tau_{A B C}$ of all states with a given $p_{\text {sum }}$.

In addition to these 'special' states, there are two classes of states that deserve additional attention. While these states have stabilizers of zero dimension [25] like generic states, these classes are also preserved by the above distillation protocols. Like the 'special' states, they extremize distillability as a function of $\tau_{A B C}$ and $p_{\text {sum }}$.

Generalized triple or Tetrahedral states. These states can be written

$$
|\psi\rangle=b|001\rangle+c|010\rangle+e|100\rangle+h|111\rangle .
$$

We are mainly interested here in the symmetric state $b=c=e$; for this case $p_{A}=p_{B}=$ $p_{C}=2 b^{2}, p_{\text {sum }} \leq 2$. This form is preserved by the steps of the infinitesimal distillation protocol, which make the coefficients evolve according to (22); the yield is easily integrated according to (29) to give $Y=2\left(2-p_{\text {sum }}\right)=4\left(1-3 b^{2}\right)$; the residual tangle is $\tau_{A B C}=$ $16 b^{3} \sqrt{1-3 b^{2}}=\sqrt{(4-Y)^{3} Y / 27}$. This yield is identical to that of the maximal slice states 
as a function of $p_{\text {sum }}$, but not as a function of $\tau_{A B C}$; from Figs. 1 and 2 , we see that they are states of minimal distillability in terms of both $p_{\text {sum }}$ and $\tau_{A B C}$.

Zero residual tangle (ZRT) states. Dür, Vidal and Cirac have shown that no states with $\tau_{A B C}=0$ can be converted to GHZ triplets with nonzero probability, so $Y=0$. They also showed that all such states can be written in the form

$$
|\psi\rangle=a|000\rangle+b|001\rangle+c|010\rangle+e|100\rangle .
$$

This is in general not in the Schmidt form of section III. These states include the triple states $a=0$ as a subclass (for which (40) is in Schmidt form). The triple states form a boundary of this set, and any ZRT state will evolve under the distillation protocol to a triple state. These states have $p_{\text {sum }} \geq 2$.

All these 'special' states have symmetries which account for both their enlarged stabilizers and their extremal distillability. One way of seeing this is to note that the various standard forms given in section II, which for generic states all require distinct bases, often coincide for these special states. For instance, the generalized GHZ states (32) are simultaneously in Schmidt, two-term, minimal, LPS and Griffiths-Niu form. ZRT and triple states cannot be written in two-term form, but can be written with three terms; the triple states (31) are simultaneously in Schmidt, minimal, three-term and Griffiths-Niu form. Slice states written in the form (35) are simultaneously in both Schmidt and LPS standard forms.

It is easiest to see how the the distillability of these states compares to that of generic states by plotting their yields $Y$ as a function of $p_{\text {sum }}$ and $\tau_{A B C}$ along with the numerical results for a large sample of randomly generated states. We have plotted these quantities in Figs. 1 and 2, with the families of 'special' states indicated. We see that most of these states are indeed special as far as distillation is concerned: they form the boundaries of the plotted regions. The quantity $\tau_{A B C}$ does seem to be closely related to distillability, as conjectured, though this relationship is not exact; for a given value of $\tau_{A B C}$ states with a range of $Y$ values exist, but the range is not very wide. This range is bounded at the top by the generalized GHZ and maximal slice states, and at the bottom by the symmetric generalized triple state. All ZRT states have $Y=\tau_{A B C}=0$. There is also a relationship between $p_{\text {sum }}$ and $Y$, though again for a given $p_{\text {sum }}$ there is a range of $Y$ values. This range too is bounded above by the generalized GHZs, and below by the ZRT states, generalized triples and maximal slice states. These upper and lower bounds are both linear; the upper bound is exactly the same as that for Bernstein and Bennett's Procrustean technique [3], reflecting the fact that generalized GHZ states can be distilled by exactly the same techniques which work in the bipartite case.

A reasonable question is to what extent these yields are artifacts of the particular distillation protocol we use. After all, this technique is only one possible way of producing GHZs, in general not the optimal method even for a single copy of a three-qubit state.

Fortunately, we can actually answer this question. Recently, Acín, Jané, Dür and Vidal 21] have discovered the optimal algorithm for transforming a single copy of a three-qubit state into a GHZ. This involves performing a POVM on each of the three bits, designed to project the states in the two-term representation onto tri-orthogonal vectors. Finding the correct POVM for an arbitrary state involves maximizing a somewhat involved function, but is easily done numerically. We have done so for a large sample of random states, as well as for the members of the 'special' classes enumerated by Carteret and Sudbery. 
The optimal yield is higher, in general, than that of the infinitesimal algorithm of section IV, though they are surprisingly close for most states. However, for the 'special' states, the yields are identical. In other words, the infinitesimal distillation technique gives the optimal yield for these classes of states. Quite remarkably, if we plot Figures 1 and 2 for the optimal GHZ distillation protocol, the figures look completely unchanged.

Thus we can see that by both the optimal and the infinitesimal techniques, these classes of special states extremize the yield of GHZs as a function of both $\tau_{A B C}$ and $p_{\text {sum. This }}$ strongly supports the conclusion that these states do indeed have unusual entanglement properties, and are worthy of further study.

\section{CONCLUSIONS}

We have examined tripartite entanglement from both an analytical and an operational point of view. In the bipartite case, which is well understood and to which we have turned for clues, the analytical and operational aspects of entanglement are closely related: the entanglement properties of a single copy are given by the locally invariant parameters, the Schmidt coefficients, which also determine their operational characteristics. We have looked for similar connections in the three-qubit case. Here at least five locally invariant parameters are required, as opposed to just one in the two-qubit case. We have examined several ways of choosing these five parameters, looking in particular at generalizations of the bipartite Schmidt decomposition. One representation in particular, the "Schmidt form," has useful properties which made it simple to prove the efficacy of the infinitesimal GHZ distillation protocol of [11]; it can also be parametrized in terms of five physically meaningful quantities.

We have looked for connections between these parameters and yields in distilling GHZ triplets, as well as connections with the residual tangle of Coffman et al. We have shown that the 'special' classes of states enumerated by the theorem of Carteret and Sudbery extremize the distillation yield as functions of the residual tangle $\tau_{A B C}$ and $p_{\text {sum }}=p_{A}+p_{B}+p_{C}$.

Although a certain amount amount of progress towards understanding tripartite entanglement has been made, at least for qubits, many important questions remain unanswered. For example, the number of states in the asymptotic minimum reversible entanglement generating set (MREGS) [20,26,27] for three-qubit states, and for tripartite states in general, is still unknown. No asymptotically reversible (or optimal but irreversible) distillation technique for GHZ states is known. The search for solutions to these and related problems is ongoing.

\section{ACKNOWLEDGMENTS}

We would like to thank H.A. Carteret, W. Dür, R.B. Griffiths, A. Sudbery and G. Vidal for many useful conversations. This work was supported by NSF Grant No. PHY-9900755. 


\section{REFERENCES}

[1] C.H. Bennett, G. Brassard, C. Crépeau, R. Jozsa, A. Peres and W.K. Wootters, Phys. Rev. Lett. 70, 1895 (1993).

[2] D. Deutsch, A. Ekert, R. Jozsa, C. Macchiavello, S. Popescu and A. Sanpera, Phys. Rev. Lett. 77, 2818 (1996).

[3] C.H. Bennett, H.J. Bernstein, S. Popescu and B. Schumacher, Phys. Rev. A 53, 2046 (1996).

[4] M.A. Nielsen, Phys. Rev. Lett. 83, 436 (1999).

[5] G. Vidal, Phys. Rev. Lett. 83, 1046 (1999); D. Jonathan and M.B. Plenio, Phys. Rev. Lett. 83, 1455 (1999).

[6] M. Horodecki, P. Horodecki and R. Horodecki, quant-ph/9607009.

[7] A.V. Thapliyal, Phys. Rev. A 59, 3336 (1999).

[8] N. Linden and S. Popescu, Fortsch. Phys. 46, 567 (1998).

[9] J. Schlienz, Ph.D. thesis.

[10] O. Cohen, unpublished.

[11] O. Cohen and T.A. Brun, Phys. Rev. Lett. 84, 5908 (2000).

[12] A. Sudbery, J. Phys. A 34, 643, (2001).

[13] A. Acín, A. Andrianov, L. Costa, E. Jané, J.I. Latorre and R. Tarrach, Phys. Rev. Lett. 85, 1560 (2000).

[14] A. Higuchi and A. Sudbery, Phys. Lett. A 273, 213 (2000).

[15] H.A. Carteret, A. Higuchi and A. Sudbery, J. Math. Phys. 41, 7932 (2000).

[16] N. Linden, S. Popescu, A. Sudbery, Phys. Rev. Lett. 83, 243 (1999).

[17] J. Kempe, Phys. Rev. A 60, 910 (1999).

[18] V. Coffman, J. Kundu and W.K. Wootters, Phys. Rev. A 61, 2306 (2000).

[19] H.A. Carteret and A. Sudbery, J. Phys. A 33, 4981 (2000).

[20] C.H. Bennett, S. Popescu, D. Rohrlich, J.A. Smolin and A.V. Thapliyal, to appear in Phys. Rev. A, quant-ph/9908073.

[21] A. Acín, E. Jané, W. Dür and G. Vidal, Phys. Rev. Lett. 85, 4811 (2000).

[22] W. Dür, G. Vidal and J.I. Cirac, Phys. Rev. A 62, 062314 (2000)

[23] A. Acín, A. Andrianov, E. Jané, R. Tarrach, quant-ph/0009107.

[24] Chi-Sheng Niu and Robert B. Griffiths, Phys. Rev. A 60, 2764 (1999).

[25] H.A. Carteret, private communication.

[26] N. Linden, S. Popescu, B. Schumacher and M. Westmoreland, quant-ph/9912039.

[27] S. Wu and Y. Zhang, quant-ph/0004020. 
Figure 1. Here we plot the primary yield of GHZ triplets from the infinitesimal distillation algorithm of section III vs. the square of the residual tangle $\tau_{A B C}{ }^{2}$ for various 'special states' as well as a random sample of generic states. We see that all states lie between two curved boundaries; the generalized GHZ and maximal slice states lie on the upper boundary, while the generalized triple states lie on the lower boundary. The triple states all have both $\tau_{A B C}$ and the yield equal to zero. Interestingly, the maximal slice states appear to be high-yield states when plotted against $\tau_{A B C}$, but low-yield when plotted against $p_{\text {sum }}=p_{A}+p_{B}+p_{C}$; for a given value of $\tau_{A B C}$ these states minimize $p_{\text {sum }}$.

Figure 2. Here we plot the primary yield of GHZ triplets from the infinitesimal distillation algorithm of section III vs. $p_{\text {sum }}=p_{A}+p_{B}+p_{C}$ for various 'special states' as well as a random sample of generic states. We see that all states lie between two linear boundaries; the generalized GHZ states lie on the upper boundary, while the maximal slice and generalized Triple states lie on the lower boundary, and the triple states are the zero-yield states between $p_{\text {sum }}=2$ and $p_{\text {sum }}=3$. The upper linear boundary corresponds to the yield of Bernstein and Bennett's Procrustean method of EPR distillation in the bipartite case. 


\section{FIGURES}

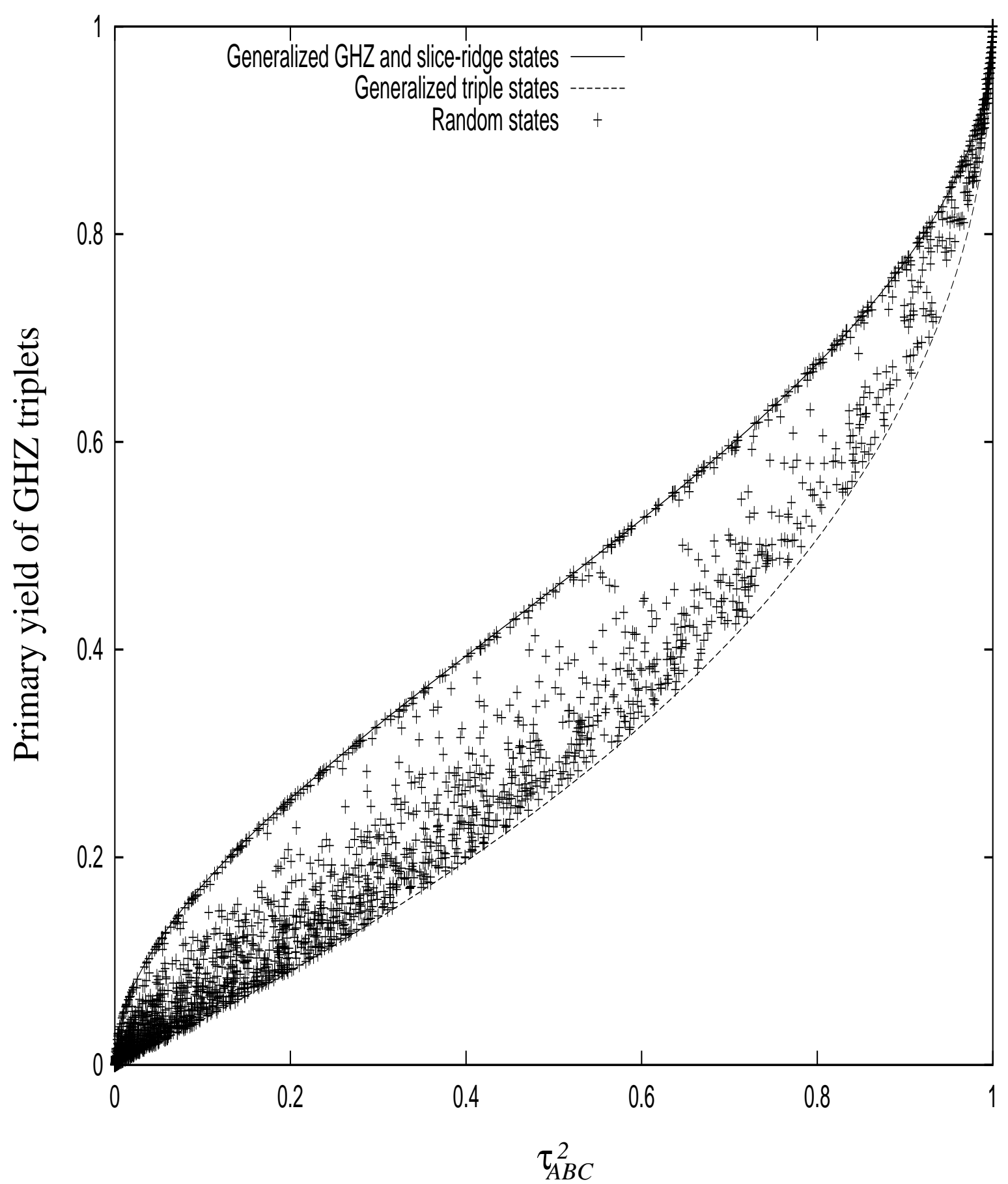

Figure 1. 


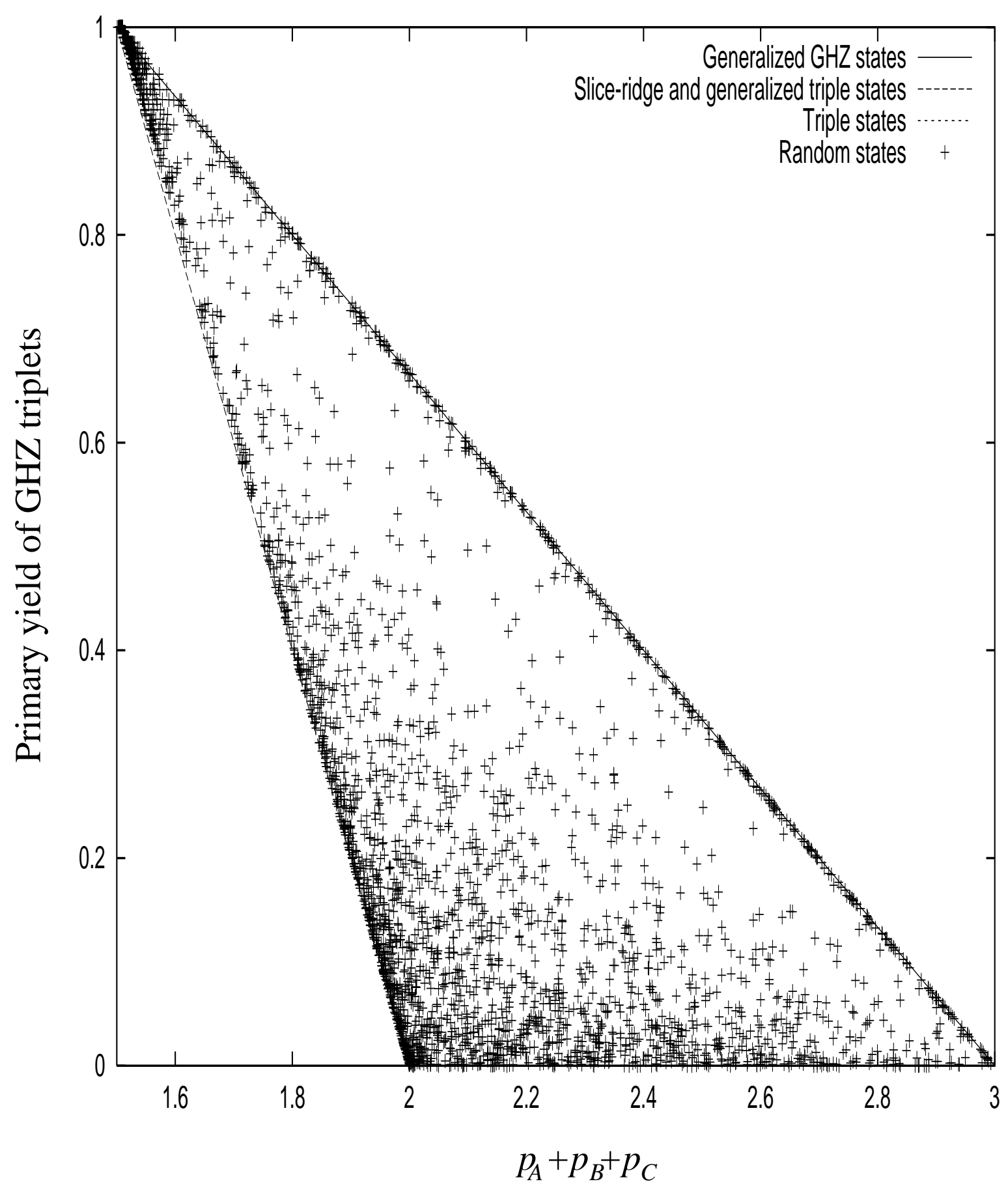

Figure 2. 